\title{
Usefulness of Luliconazole 1\% and Salicylic Acid 3\% as FDC Cream in the Treatment of Hyperkeratotic Tinea Pedis
}

\author{
Dhoot D*, Deshmukh G, Mahajan H, Rashmi KM and Barkate H \\ Global Medical Affairs (IF), Glenmark Pharmaceuticals Ltd, India
}
*Corresponding author: Dr. Dhiraj Dhoot, Glenmark pharmaceuticals Ltd, B D Sawant Marg, Chakala, Andheri (E), Mumbai 400099, India, Tel: +919619811219; Email: Dhiraj.dhoot@ glenmarkpharma.com

\begin{abstract}
glenmarkpharma.com
\end{abstract}
\section{Research Article}

Volume 5 Issue 4

Received Date: November 11, 2020

Published Date: November 25, 2020

DOI: $10.23880 /$ cdoaj-16000225

\section{Abstract}

Background: Tinea Pedis is one of the most common dermatophytosis and sometimes presents with moccasin or hyperkeratotic Tinea Pedis. Some topical antifungals are effective; however, long duration of therapy is required, which often reduce the treatment compliance of patients.

Methods: To seek insights on short period therapy in hyperkeratotic Tinea Pedis, we conducted a multicentre, retrospective analysis of data collected from 36 dermatology clinics to study effectiveness and safety of Luliconazole $1 \%$ and Salicylic acid (SA) $3 \%$ as FDC cream applied for 2 weeks.

Results: A data of 109 patients' were included in this analysis. All patients have received some of the topicals and switched to FDC. Fifty eight patients received FDC as once a day (Group 1) and 51 patients as twice a day (Group 2). The clinical improvement rate (percentage of 'marked improvement' plus `moderate improvement') was $>60 \%$ after 2 weeks of treatment in both the groups. Patients in both the groups tolerated therapy very well. In group I, 15 patients (25.8\%) experienced $\mathrm{AE}$ and in group II, 32 patients (62.7\%) reported AE. Majority of AE were mild in nature and none of the patients discontinued treatment in entire treatment duration. But significant difference was noted between two groups in terms number of $\mathrm{AE}$ $(\mathrm{p}<0.05)$.

Conclusion: Hyperkeratotic Tinea Pedis is more recalcitrant to treatment due to thick scales leading to ineffectiveness of topical antifungals. The short combination therapy with luliconazole and salicylic acid has been found to be effective and safe. Though it is one of the valuable options for hyperkeratotic Tinea Pedis, but it should be prescribed as once a day only to minimize AE.

Keywords: Luliconazole; Salicylic acid; Hyperkeratotic Tinea Pedis; Real world; India

\section{Introduction}

Tinea Pedis is one of the most common dermatophytosis and sometimes presents with moccasin or hyperkeratotic Tinea Pedis, which usually affects the entire plantar surface and sides of the foot [1-3]. Patients with hyperkeratotic Tinea Pedis present with a chronic "dry type" infection and thick, hyperkeratotic scale. The thick scale on the plantar surface of the foot usually impedes the absorption of the antifungal agent leading infection recalcitrant to topical anti-fungal therapy alone [4]. Consequently, adjunctive oral therapy for 1 to 4 weeks is often necessary, thereby contributing to the potential adverse effects and interactions of the drug $[2,5]$.

In search for better topical treatment of recalcitrant moccasin Tinea Pedis, the characteristic thick scale must be 


\section{Clinical Dermatology Open Access Journal}

addressed. Topical keratolytic, such as $3 \%$ salicylic acid, is a practical approach to address this problem. By increasing desquamation process and augmenting the shedding of scale, topical salicylic acid (SA) reduces fungal load. Through this keratolytic mechanism, topical SA may work synergistically with and increase the efficacy of topical antifungals in the treatment of hyperkeratotic Tinea Pedis. Several reports have shown the usefulness of SA in the management of hyperkeratotic Tinea Pedis [6-8].

Topical luliconazole is a broad-spectrum anti-fungal agent with inhibitory activity against pathogenic dermatophytes. In addition, it is most potent topical antifungal with lowest MIC as compared to other topical anti-fungal [9-10].

Recently, Fixed Drug Combination (FDC) of luliconazole $1 \%$ and SA $3 \%$ were commercialized in Indian pharmaceutical market for the management of hyperkeratotic Tinea Pedis. The objective of this study is to assess the clinical utility of this FDC in real world settings.

After obtaining ethics committee approval (Suraksha ethics committee Reg. ECR/644/Inst/MH/2014/RR-17), a retrospective analysis of data from medical records was carried out at 36 centers across India, to study the effectiveness and tolerability of this FDC in the management of hyperkeratotic Tinea Pedis. Only those patients' records were considered who were diagnosed as hyperkeratotic Tinea Pedis, were in the age group of 18-60 years and were prescribed FDC of 1\% luliconazole and 3\% salicylic acid. Hyperkeratotic Tinea Pedis patients treated with other antifungal agents in combination with this FDC were excluded.

According to patients' clinical records, assessments were done at baseline and 2 weeks for effectiveness with 5 step scale as; marked improvement= improvement in symptom Score by $>90 \%$; moderate improvement= improvement in symptom Score by $>50 \%$ but $<90 \%$; slight improvement= improvement in symptoms Score by $<50 \%$; no improvement= no change in symptom score and worsening= aggravation of symptom score. Considering the adverse events (AE), we rated the safety of FDC in accordance with the following 4-step scale: $1=$ No adverse events; $2=$ almost safe with mild $\mathrm{AE} ; 3=$ minor problems with the safety (moderate $\mathrm{AE}$ ); and $4=$ not safe (severe $\mathrm{AE}$ leading to discontinuation).

At the completion of the study, the usefulness of the treatment was evaluated combining the overall effectiveness ratings and the safety ratings and in accordance with the following 5-step scale: $1=$ =markedly useful; $2=$ useful; $3=$ slightly useful; $4=$ not useful; and $5=$ harmful as shown in table 1. Binomial variables were expressed as number \& percentage and continuous variables as mean (S.D.). Paired $t$ test was used for comparisons between baseline and followup measurements and significant difference was defined at a level of $\mathrm{p}<0.05$.

\begin{tabular}{|c|c|c|}
\hline Markedly Useful & $\begin{array}{c}\text { Effectiveness } \\
\text { Marked } \\
\text { improvement }\end{array}$ & Safety \\
\hline Useful & $\begin{array}{c}\text { Moderate } \\
\text { improvement }\end{array}$ & $\begin{array}{c}\text { No AE + Mild/Mod } \\
\mathrm{AE}\end{array}$ \\
\hline Slightly useful & $\begin{array}{c}\text { Slight } \\
\text { improvement }\end{array}$ & $\begin{array}{c}\text { No AE + Mild/Mod } \\
\mathrm{AE}\end{array}$ \\
\hline Not useful & No change & Moderate + severe AE \\
\hline Harmful & Worsening & Moderate + severe AE \\
\hline
\end{tabular}

Table 1: Clinical usefulness rating.

A total of 109 patients' data were included in this analysis. Two groups were identified based on dosage of frequency. Group I received FDC as once a day dosing while group II received FDC as twice a day regime. Baseline demographics are described in table 2 .

\begin{tabular}{|c|c|c|c|}
\hline Lulican S Dosage frequency & OD & BD & p value \\
\hline $\mathrm{N}$ & 58 & 51 & \\
\hline $\mathrm{M}$ & 41 & 33 & \\
\hline $\mathrm{F}$ & 17 & 18 & 0.31 \\
\hline Age (years) & $35.16 \pm 10.27$ & $37.27 \pm 10.22$ & 0.23 \\
\hline Duration of disease (Days) & $30.2 \pm 5.6$ & $27.8 \pm 5.2$ & \\
\hline Amorolfine & 6 & 4 & \\
\hline Ciclopirox & 13 & 3 & \\
\hline Eberconazole & 2 & 2 & \\
\hline Ketoconazole & 3 & 17 & \\
\hline Luliconazole & 18 & 5 & \\
\hline Sertaconazole & 4 & & \\
\hline
\end{tabular}




\section{Clinical Dermatology Open Access Journal}

\begin{tabular}{|c|c|c|c|}
\hline Terbinafine & 4 & 3 & \\
\hline Naïve & 8 & 6 & \\
\hline \multicolumn{4}{|c|}{ Disease severity } \\
\hline Moderate & 14 & 14 & \\
\hline Severe & 44 & 37 & \\
\hline \multicolumn{4}{|c|}{ Baseline symptom score } \\
\hline Erythema & $2.52 \pm 0.76$ & $2.00 \pm 0.89$ & 0.27 \\
\hline Scaling & $2.50 \pm 0.71$ & $2.62 \pm 0.85$ & 0.4 \\
\hline Pruritus & $2.46 \pm 0.76$ & $2.30 \pm 0.82$ & 0.63 \\
\hline TSS & $7.48 \pm 1.91$ & $6.91 \pm 1.86$ & 0.38 \\
\hline
\end{tabular}

Table 2: Baseline demographics.

At day 14, in terms of total symptom score; there was statistical difference in both the groups compared to baseline as shown in figure 1. But no statistical difference was noted between two groups $(p=0.25)$. Statistical difference $(p<0.05)$ was noted for all individual symptoms in both the groups at day 14 compared to baseline (Figure 2). But no statistical difference was noted between both the groups except for erythema (Table 3) at day 14.
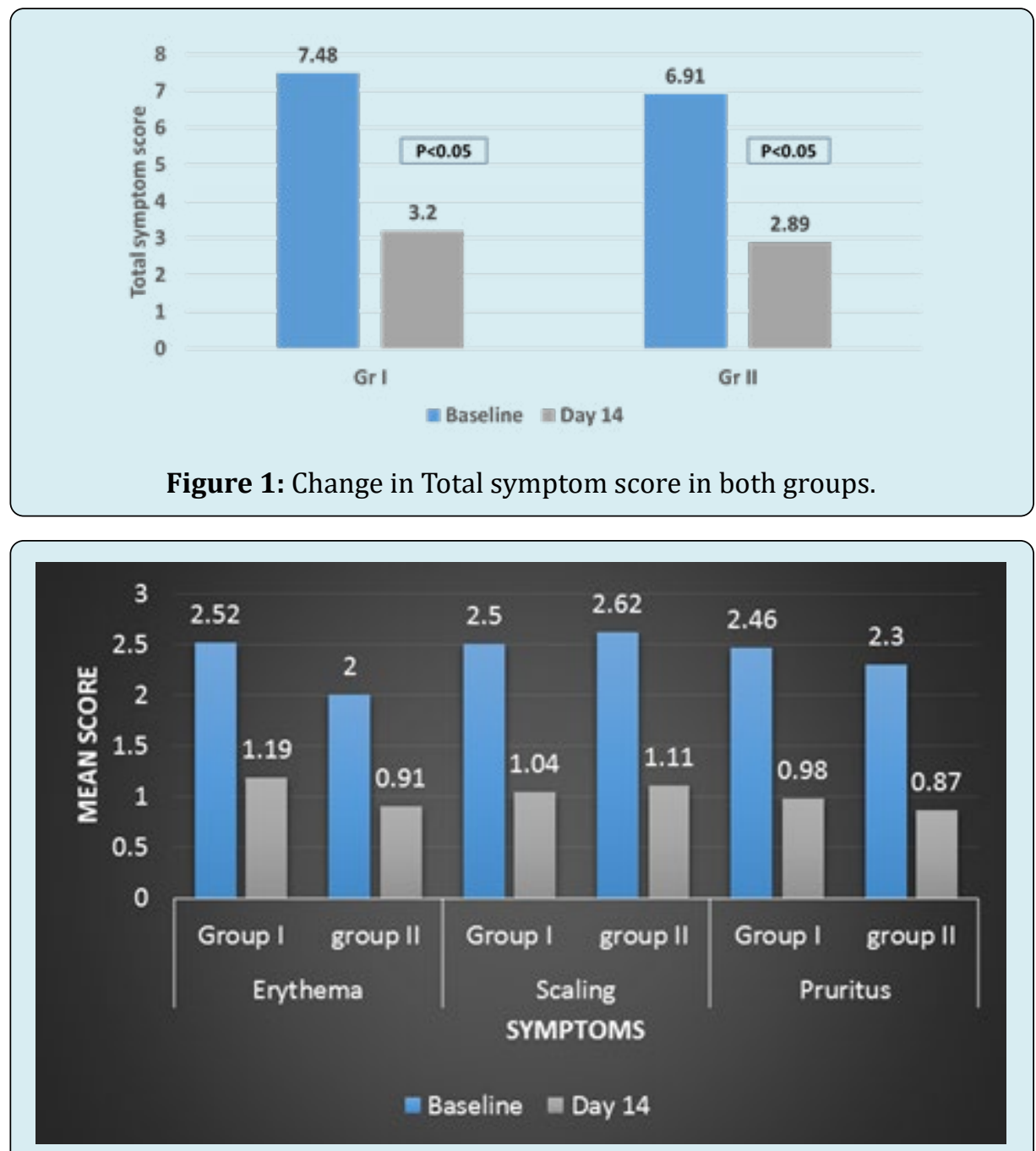

Figure 2: Change in individual symptom score in both groups. 


\section{Clinical Dermatology Open Access Journal}

Patients in both the groups tolerated therapy very well. In group I, 15 patients (25.8\%) experienced $\mathrm{AE}$ and in group II, 32 patients (62.7\%) reported AE. Majority of AE were mild in nature and none of the patients discontinued treatment in entire treatment duration (Table 3). But significant difference was noted between two groups in terms of AE $(p<0.05)$.

\begin{tabular}{|c|c|c|c|}
\hline Symptom score & Group I & Group II & p value \\
\hline Erythema & $1.19 \pm 0.47$ & $0.91 \pm 0.53$ & $<0.05$ \\
\hline Scaling & $1.04 \pm 0.54$ & $1.11 \pm 0.58$ & 0.6 \\
\hline Pruritus & $0.98 \pm 0.52$ & $0.87 \pm 0.56$ & 0.38 \\
\hline TSS & $3.20 \pm 1.21$ & $2.89 \pm 1.37$ & 0.25 \\
\hline Adverse effects & Group I & Group II & \multirow{2}{*}{ p value } \\
\hline No AE & 43 & 19 & $<0.05$ \\
\hline Mild & 14 & 24 & 8 \\
\hline
\end{tabular}

Table 3: Group-wise effectiveness and safety assessment at Day 14.

Based on clinical usefulness rating $36 / 58$ patients (62\%) in group I and 30/51 (60\%) in group II were classified under markedly useful and useful category respectively (Tables 4 $\& 5)$.

\begin{tabular}{|c|c|c|c|c|c|}
\hline Effectiveness rating & $\begin{array}{c}\text { Marked } \\
\text { improvement }\end{array}$ & $\begin{array}{c}\text { Moderate } \\
\text { improvement }\end{array}$ & Slight improvement & No change & Worsening \\
\hline Group 1 (N) & 8 & 28 & 15 & 5 & 2 \\
\hline Group 2 (N) & 8 & 22 & 14 & 4 & 3 \\
\hline
\end{tabular}

Table 4: Effectiveness of FDC.

\begin{tabular}{|c|c|c|c|c|c|}
\hline Usefulness rating & Markedly Useful & Useful & Slightly useful & Not useful & Harmful \\
\hline Group 1 (N) & 8 & 28 & 15 & 5 & 2 \\
\hline Group 2 (N) & 8 & 22 & 14 & 4 & 3 \\
\hline
\end{tabular}

Table 5: Clinical usefulness of FDC.

The goal of treatment in hyperkeratotic Tinea Pedis is complete symptom control. But, anti-fungal are not expected to heal hyperkeratotic type Tinea Pedis with only topical applications, because of the poor penetration into the affected area. Consequently, an oral administration of anti-fungal is required. However, because of side-effects and drug interactions with concomitant drugs, such oral antifungal are not necessarily appropriate for all patients. Thus, combination therapy of anti-fungal and keratolytic is often needed in daily practice in the management of hyperkeratotic Tinea Pedis [11]. In India, SA is commonly used as keratolytic agent in the management of hyperkeratotic Tinea Pedis since many years.

Currently, there are no studies regarding combination therapy of luliconazole and SA but there are many studies suggesting usefulness of anti-fungal and keratolytic in the management of hyperkeratotic Tinea Pedis. Tanuma, et al. [11] reported that combination therapy of $1 \%$ lanoconazole and $10 \%$ urea was highly effective and safe and should be recommended for patients with hyperkeratotic Tinea Pedis in whom an oral therapy is not appropriate or a sufficient improvement with oral medications cannot be expected [11].

In one study by Tanuma, et al. [12], it was found that combination therapy with bifonazole and $10 \%$ urea was beneficial in $83 \%$ of the patients. Similarly, Elewski, et al. [1] reported that, all patients were free of dermatological symptoms after 2-3 weeks of combination therapy with $40 \%$ urea cream and ciclopirox cream. But in all these studies, both the products were used separately and not as FDC. Secondly, in all studies urea was used as keratolytic agent and for 12 weeks. In our study as well, at the end of 2 weeks, $62 \%$ and $60 \%$ of the patients were under the category of markedly useful and useful in group I and group II respectively. But since SA was keratolytic agent in our study, results of these studies can't be compared with our studies. 
One of the most common adverse effect of SA is irritation which is due to its strong acid action. Secondly, severe burning sensation may occur due to application of SA over the inflamed area [8]. In one study by Saoji, et al. [8], in spite of burning sensation in all patients, effectiveness was seen in $88 \%$ of the patients. There are many reports regarding safety of topical salicylic acid $[13,14]$ but most of the applications were in other formulation and for short contact. As per one review [15] on toxicity from topical salicylic acid, it was concluded that in last 50 years, there were total 25 cases of toxicity with topical use of $6 \%$ salicylic acid and over with as little as $40 \%$ body surface area.

In our study, in group I, 25.8\% patients reported AE where as in group II, $62.7 \%$ patients reported the same. Though all AE were AE mild in nature, it is to conclude that luliconazole and salicylic acid combination therapy can cause some amount of irritation and this should be explained to patients before starting of therapy for better treatment adherence. Secondly, this FDC should be prescribed as once daily application to minimize the irritation.

\section{Limitation \& Conclusion}

To the best of our knowledge, the present study is the first real world experience with use of this FDC (luliconazole $1 \%$ and SA 3\%) in the management of hyperkeratotic Tinea Pedis in India. The only major shortcoming of the present study is its nature, i.e. retrospective real world data analysis. Despite this limitation, it is interesting to point out that the clinical response of this FDC was enhanced in patients with short course of therapy. Therefore, topical treatment with the FDC of luliconazole 1\% and SA 3\% may be a good addition to the early part of management algorithm of hyperkeratotic Tinea Pedis.

\section{References}

1. Elewski BE, Haley HR, Robbins CM (2004) The use of $40 \%$ urea cream in the treatment of moccasin Tinea Pedis. Cutis 73(5): 355-357.

2. Richardson M, Elewski B (2000) Superficial Fungal Infections. Oxford: Health Press Limited.

3. Kemna ME, Elewski BE (1996) A U.S. epidemiologic survey of superficial fungal diseases. J Am Acad Dermatol 35: 539-542.

4. Leyden JL (1994) Tinea Pedis pathophysiology and treatment. J Am Acad Dermatol 31(3): S31-S33.

5. Rupke SJ (2000) Fungal skin disorders. Prim Care 27(2): 407-421.
6. Rajagopalan M, Inamadar A, Mittal A, Miskeen AK, Srinivas CR, et al. (2018) Expert Consensus on the Management of Dermatophytosis in India (ECTODERM India). BMC Dermatol 18(1): 6.

7. Sardana K, Mahajan K, Mrig PA (2017) Fungal Infections: Diagnosis and treatment. $1^{\text {st }}$ (Edn.), CBS Publishers and Distributors, pp: 266-317.

8. Saoji V, Madke B (2019) Efficacy of salicylic acid peel in dermatophytosis. Indian J Dermatol Venereol Leprol.

9. Rudramurthy SM, Shankarnarayan SA, Dogra S, Shaw D, Mushtaq K, et al. (2018) Mutation in the Squalene Epoxidase Gene of Trichophyton interdigitale and Trichophyton rubrum Associated with Allylamine Resistance. Antimicrob Agents Chemother. 62(5): e02522-17.

10. Das S, De A, Saha R, Sharma N, Khemka M, et al. (2020) The current Indian epidemic of dermatophytosis: A study on causative agents and sensitivity patterns. Indian J Dermatol 65: 118-122.

11. Tanuma H, Tanuma M, Abe M, Kume H (2001) Usefulness of lanoconazole (Astat) cream in the treatment of hyperkeratotic type Tinea Pedis. Comparative study of monotherapy and combination therapy with $10 \%$ urea ointment (Pastaron). Mycoses 44(5): 181-190.

12. Tanuma H, Doi $M$, Sato N, Nishiyama $S$, Abe $M$, et al. (2000) Bifonazole (Mycospor cream) in the treatment of moccasin-type Tinea Pedis. Comparison between combination therapy of bifonazole cream $+10 \%$ urea ointment (Urepearl) and occlusive dressing therapy with the same agents. Mycoses 43(3-4): 129-37.

13. Taylor JR, Halprin KM (1975) Percutaneous absorption of salicylic acid. Arch Dermatol 111(6): 740-743.

14. Bari AU, Iqbal Z, Rahman SB (2005) Tolerance and safety of superficial chemical peeling with salicylic acid in various facial dermatoses. Indian J Dermatol Venereol Leprol 71(2): 87-90.

15. Madan RK, Levitt J (2014) A review of toxicity from topical salicylic acid preparations. J Am Acad Dermatol 70(4): 788-792. 